\title{
The Antimicrobial Activity of Silk Fabrics Dyed with Jasmine Tea and Jasmine Petal Tea Extracts
}

\author{
Jin-Soun Jung, Bo-Hee Lee, Keum-Hi Mun and Ja-Young Kwon \\ Department of Fashion Design, Semyung University, Jecheon 390-711, Korea \\ bobejin@semyung.ac.kr
}

\begin{abstract}
The purpose of this study is to develop a natural functional material with insecticidal properties and antimicrobial activity for the application of emotional robot development for the early treatment use of children with autism spectrum disorder. As a part of the study, I investigate the antimicrobial activity of silk fabrics dyed with jasmine tea and jasmine petal tea extracts. Bacteria used for the test of antimicrobial activity were Staphylococcus aureus ATCC 6538 and Klebsiella pneumoniae ATCC 4352. The presence of phenol, known as an antimicrobial component, was measured from jasmine tea and jasmine petal tea extracts. UVVis analysis and FT-IR analysis were also employed. Silk fabrics dyed with jasmine tea extracts appeared to have significant antimicrobial activity of $99.9 \%$ for $S$. aureus, regardless of dyeing concentration. On K. pneumoniae, silk fabrics dyed with stock solution and one-half-fold diluted solution of jasmine tea extracts presented significantly high antimicrobial activity of 99.9\%. In contrast, no antimicrobial activity was observed from all silk fabrics dyed with jasmine petal tea extracts, regardless of the type of bacteria. On $S$. aureus following 20 hours of irradiation, silk fabrics dyed with jasmine tea extracts presented high antimicrobial activity of 93.3\%, regardless of dyeing concentration. On K. pneumoniae after 20 hours of irradiation, only the silk fabric dyed with a stock solution of jasmine tea extracts appeared to have any antimicrobial activity (40.6\%). On S. aureus after dry cleaning, silk fabrics dyed with jasmine tea extract had significantly higher antimicrobial activity of 99.9\%, identical to its effectiveness before dry cleaning, regardless of the dyeing concentration. On K. pneumoniae after dry cleaning, silk fabrics dyed with stock solution and one-half-fold diluted solution of jasmine tea extracts had significantly higher antimicrobial activity of $99.9 \%$, identical to their effectiveness before dry cleaning. The total phenol content was higher in the jasmine tea than in the jasmine petal tea. From the results of UV-Vis analysis, the ethanol extraction solution of jasmine tea and jasmine petal tea had absorption maximums of $\lambda$ max at $272 \mathrm{~nm}$ and $277 \mathrm{~nm}$ ( $\lambda$ max of tannin is 272-285 $\mathrm{nm}$ ), respectively. From the results of FT-IR analysis, jasmine tea and jasmine petal tea showed three main common absorption bands in the 1750-700 $\mathrm{cm}^{-1}$ range: 1615-1606, 1518-1507, and 1043$1030 \mathrm{~cm}^{-1}$. Therefore, it was able to be determined that the form of tannin obtained in jasmine tea and jasmine petal tea is a kind of hydrolyzable tannin.
\end{abstract}

Keywords: anti-microbial activity, silk, dyeing, extract, jasmine tea, jasmine petal tea

\section{Introduction}

The material from which most existing emotional robots are constructed is cold, hard plastic or synthetic fur. The animal emotional robots constructed from plastic material do not help in the treatment of children with developmental disabilities because the users can not feel peace when interacting with the robot, due to the characteristics of the material from 
which it is constructed. Also, even if faux fur has the feel of real animals, users cannot avoid problems associated with respiratory allergic symptoms such as asthma due to house dust mites, chronic rhinitis, bronchitis, atopic dermatitis, conjunctivitis, and chronic eczema through the interaction of the user with the robot. Therefore, the development of a functional material is necessary that has insecticidal effects on house dust mites and antimicrobial activity on bacteria to protect the emotional robots' users from the harmful environments present in daily life. Recently, the problem has been identified that synthetic insecticidal agents and synthetic antimicrobial agents used to treat fabric to inhibit the growth of bacteria gradually wear off of the fabric and can cause skin disorders. Furthermore, another problem is that the synthetic insecticidal agents and synthetic antimicrobial agents used to treat fabric gradually fade by repeated washing and, due to their presence in the washing water, can lead to environmental pollution. Therefore, it is urgent to develop a natural functional material that can replace the chemically-treated materials currently in use. The term 'herb' means 'grass' and is derived from the Latin 'herba'. Herbs were used for the first time by the ancient Greek scholar Theophrastos. Over 2,500 species of native herbs have been identified [1]. Several culinary herbs and spices are now known to have beneficial effects for human health, with properties including digestive stimulation, anti-inflammatory, antimicrobial, antioxidant and anticarcinogenic activities [2-4], which are attributed to the predominant polyphenol compounds in these plant materials. Moreover, the volatile constituents (essential oils) that are the main cause for the use of these plants can significantly contribute to their biological activity [5]. Certain aromatic herbs are also listed as "medicinal plants" and are believed to provide the organism with extra antioxidant compounds, improve digestion, and have some antibacterial, anti-inflammatory, antiviral and anti-carcinogenic activities [6]. Phenolic acids, flavonoids, sterols and coumarins are the most-studied bioactive compounds in aromatic herbs with functional properties [6]. Herbs were widely used for the treatment of relatively mild mental neurological disorders such as depression, insomnia, headaches, and anxiety, as well as other maladies including acne, seizures, gum diseases, and gynecological diseases [79]. Interest in the functionality of herbs associated with combating bacteria and insects has increased in recent years. Accordingly, attempts to use herbs as natural antimicrobial agents have been made. Unfortunately, minimal studies of the insecticidal effects and antimicrobial activity of fabrics dyed with herbal extracts have been conducted. Additionally, studies investigating its use as a robotic material are almost nonexistent. The purpose of this study is to develop a natural functional material with insecticidal effects and antimicrobial activity for emotional robot development for the early treatment use of children with autism spectrum disorder. As a part of the study, the antimicrobial activity of silk fabrics dyed with jasmine tea and jasmine petal tea extracts was measured. First, jasmine tea and jasmine petal tea were extracted with ethanol and concentrated. Silks were dyed with the concentrate, and the dyed silks were measured for their antimicrobial activity with respect to bacteria of two kinds: Staphylococcus aureus ATCC 6538 (S. aureus) and Klebsiella pneumoniae ATCC 4352 (K. pneumoniae). The antimicrobial activity of dyeing fabrics after irradiation and dry cleaning was also measured. Additionally, the total phenol contents were measured to determine the concentration of antimicrobial compounds contained in jasmine tea and jasmine petal tea. UV-Vis analysis and FT-IR analysis were also employed.

\section{Experimental Materials and Methods}

\subsection{Experimental Materials}

Jasmine tea and jasmine petal tea was purchased from Homerose Korea (http://www.homerose.co.kr/) online. Silk fabric used for dyeing was purchased from 
Testfabrics, Inc. (Silk Adjacent Single Fiber Fabric ISO 105F06). The characteristics of the silk fabric were as follows: warp density, 135 threads/cm; weft density, 98 threads/cm; weight, $52.5 \mathrm{~g} / \mathrm{m}^{2}$; warp yarn, 30 den $\times$ ply; weft yarn, 20 den $\times$ ply; and plain weave.

\subsection{Experimental Methods}

2.2.1. Extraction: Jasmine tea and jasmine petal tea were extracted by being soaked in their 10 -fold amount of ethanol for 24 hours at room temperature and sequentially filtered. This procedure was repeated 2 times. The liquor extracts were concentrated as much as the amount of the jasmine tea and jasmine petal tea used for extraction at $40^{\circ} \mathrm{C}$ under reduced pressure using a vacuum rotator evaporator.

2.2.2. Total Phenol Content: The samples for UV-Vis analysis were diluted with $70 \%$ ethanol and filtered. The filtered extracts were used as test liquids. Gallic acid was dissolved in $70 \%$ ethanol and used as the standard solution. Absorption at $765 \mathrm{~nm}$ was read versus a prepared blank. The total phenol content of jasmine tea and jasmine petal tea was expressed as milligrams of gallic acid equivalents per gram of dry weight (mg GAE/g DW) from a calibration curve with gallic acid.

2.2.3. UV-Vis Spectra: $1 \mathrm{~g}$ dried jasmine tea and jasmine petal tea was added to $50 \mathrm{~m} \ell$ ethanol, and they were extracted at room temperature for 24 hours, and filtered. The filtered extracts were used as samples for UV-Vis analysis. The measurement of the UV-absorption characteristics was conducted in the range of 280-800 nm by using an ultraviolet spectrophotometer (JASCO V-530).

2.2.4. FT-IR Spectra: IR characteristics of jasmine tea and jasmine petal tea were analyzed by using a JASCO 4100 FT-IR spectrometer (signal averaging 32 scans at a resolution of $\left.4 \mathrm{~cm}^{-1}\right)$.

2.2.5. Dyeing: The above concentrate was used as the stock solution (100\%). The solutions were diluted to $1 / 2(50 \%)$ and $1 / 4(25 \%)$ of stock solution (100\%) including stock solution and were used as dyeing liquors. The silk fabrics were dyed at a liquor ratio of 1:100, dyeing temperature $67^{\circ} \mathrm{C}$, and dyeing time $60 \mathrm{~min}$. After dyeing, the fabrics were rinsed with cold water and dried at room temperature.

2.2.6. Irradiation Test: An irradiation test of the dyed fabrics was performed using a xenon arc lamp according to KS K ISO 105 B02:2010.

2.2.7. Dry Cleaning Test: A dry cleaning test of the dyed fabrics was performed by using the perchloroethylene component of dry cleaning solvent according to KS K ISO 3175-2.

2.2.8. Antimicrobial Activity: Bacteria used for antimicrobial activity analyses included Staphylococcus aureus ATCC 6538 (S. aureus) and Klebsiella pneumoniae ATCC 4352 (K. pneumoniae). The antimicrobial activity on these two forms of bacteria of the dyed fabrics was measured by KS K 0693:2011.

\section{Results}

Tannins are an astringent vegetable product found in a wide variety of plant components such as bark, wood, fruit, fruit pods, leaves, roots and plant galls. Tannins are defined as naturally occurring water-soluble polyphenolic compounds of high molecular weight (approximately 500-3000) containing phenolic hydroxyl groups to enable them to form effective crosslinks between proteins and other macromolecules [10]. Tannin consumption 
had shown to be beneficial for human [11] and animal health due to their bactericidal [12-13], antioxidant [14-15], nematocidal [16-17] and insecticidal [18-19] properties. Tannins are broadly grouped into two classes, hydrolysable and condensed tannins, both derived from plants. Hydrolysable tannins are secondary metabolites widespread throughout the plant kingdom. They are formed by esters of gallic acid, glucose, and products of their oxidative reactions. Among the hydrolysable tannins, the gallotannins hydrolyze to yield gallic acid, while ellagitannins hydrolyze to yield ellagic acid. Ellagitannins are the products of oxidation, leading to $\mathrm{C}-\mathrm{C}$ linkages between suitably oriented galloyl residues of glucogalloyl molecules that form hexahydroxydiphenoyl units [20-21]. The distribution of gallotannins is limited in nature, while ellagitannins are widespread in many plant families. Condensed tannins (proanthocyanidins) are derivatives of catechin and esters of gallic acid with quinic acid [22]. Jasmine green tea is known to be an excellent source of natural polyphenol antioxidants [23]. Various studies have reported that leafy herbal tea extracts exert beneficial effects on lifestyle-related diseases due to their antioxidant and antimicrobial activities [24]. It is known that the antioxidant and antimicrobial activities of leafy herbal teas are attributed to the phenolic compounds they contain [25]. Phenolic compounds include phenolic acids (hydroxybenzoic and hydroxycinnamic acids), polyphenols (hydrolyzable and condensed tannins), and flavonoids. Therefore, in this study, total phenol content, UV-Vis spectra and FT-IR spectra analyses were performed to identify whether jasmine tea and jasmine petal tea used in this study contained antimicrobial components such as tannin, a type of phenolic compound.

\subsection{Total Phenol Content}

The total phenol contents of the extracts are shown in Table 1. Here, the total phenol contents of jasmine tea and jasmine petal tea presented as 13,364.7 ppm and 7907.5 ppm, respectively. Thus, the total phenol content was higher in jasmine tea than in jasmine petal tea.

Table 1. Contents of Total Polyphenol in Jasmine Tea and Jasmine Petal Tea

\begin{tabular}{l|c}
\multicolumn{1}{c|}{ Sample } & Polyphenol Content $(\mathrm{mg} / \mathrm{kg}=\mathrm{ppm})$ \\
\hline \hline Jasmine Tea & 13364.7 \\
\hline Jasmine Petal Tea & 7907.5 \\
\hline
\end{tabular}

\subsection{Spectroscopic Analysis}

3.2.1. UV-Vis Spectra: Generally, the absorption of natural phenols is affected by many factors including the extraction solvent used, $\mathrm{pH}$, and other influences. These compounds usually have absorption maximums of $\lambda \max$ at $220-280 \mathrm{~nm}$ and can be shown to have more absorption peaks according to the number of benzene rings connected to the carbon chain [26]. Figure 1 shows the UV-VIS spectra of the ethanol extraction solution of jasmine tea and jasmine petal tea. As shown by Figure 1 and Table 2, the ethanol extraction solution of jasmine tea and jasmine petal tea had absorption maxima $\lambda \max$ at $272 \mathrm{~nm}$ and $277 \mathrm{~nm}$, respectively. Therefore, these were considered as the absorption maxima, $\lambda \max$, of tannin. 


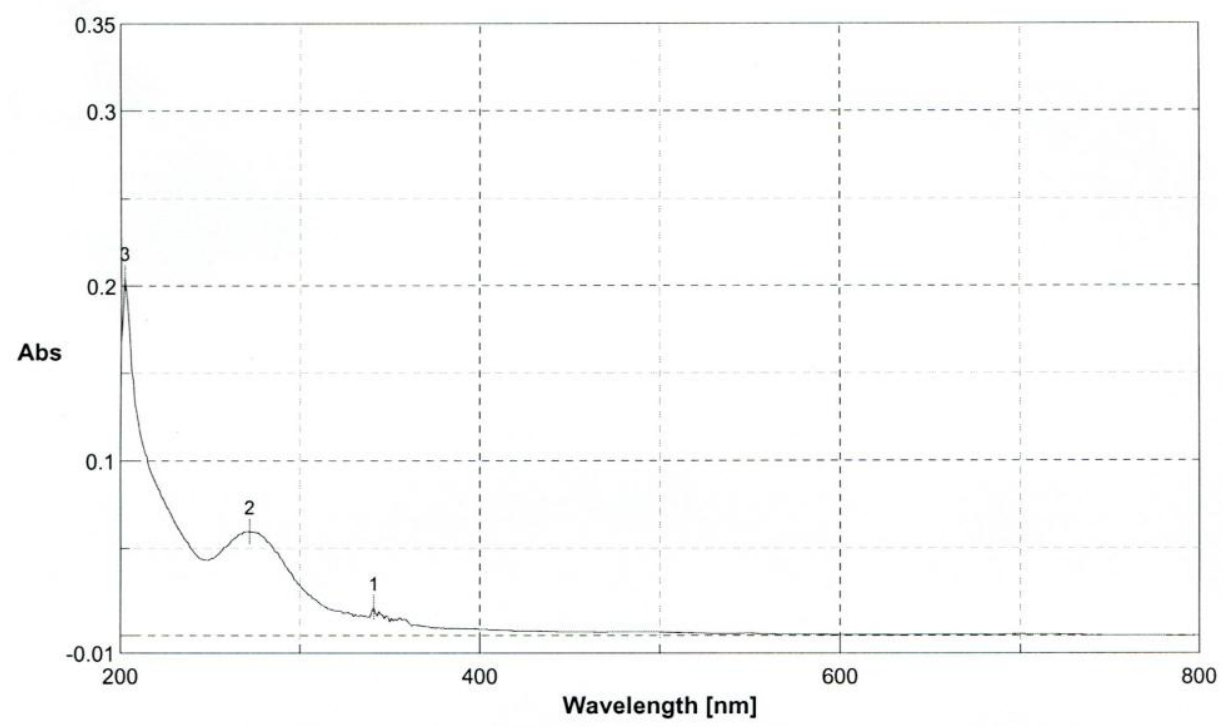

(A)

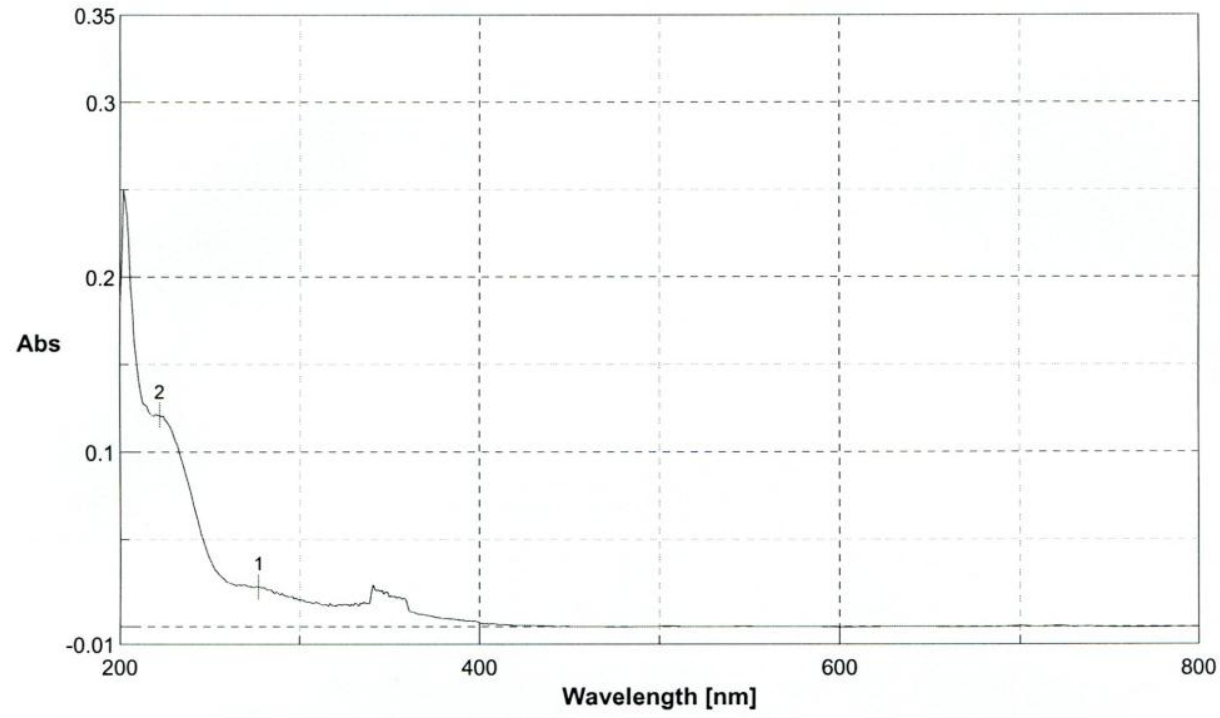

(B)

Figure 1. UV-VIS Spectra of Ethanol Extraction Solution of Jasmine Tea (A) and Jasmine Petal Tea (B)

Table 2. Wavelength and Absorption of Jasmine Tea and Jasmine Petal Tea

\begin{tabular}{c|c|c}
\hline Samples & Wavelength (nm) & Absorption \\
\hline \hline \multirow{2}{*}{ Jasmine Tea } & 202 & 0.20483 \\
\cline { 2 - 3 } & 272 & 0.05976 \\
\cline { 2 - 3 } & 341 & 0.01614 \\
\hline
\end{tabular}




\begin{tabular}{l|c|c}
\hline Jasmine Petal Tea & 222 & 0.12094 \\
\cline { 2 - 3 } & 277 & 0.02280 \\
\hline
\end{tabular}

3.2.2. FT-IR Spectra: Figure 2 shows FT-IR characteristic bands of jasmine tea and jasmine petal tea and Table 3 presents the principal bands of the FT-IR spectra. As shown by Figure 2, in this study, the region $1750-700 \mathrm{~cm}^{-1}$ was considered to be the most informative and was carefully examined. All showed three main common absorption bands in the $1750-700 \mathrm{~cm}^{-1}$ range; 1615-1606, 1518-1507, and 1043-1030 $\mathrm{cm}^{-1}$ (Figure 2). All hydrolysable tannins presented bands at 1731-1704 and 1325-1317 $\mathrm{cm}^{-1}$. Results agreed well with literature data, showing that tannins from different vegetable sources present characteristic common bands: two strong bands, one of them at $1615-1606 \mathrm{~cm}^{-1}$ was assigned to aromatic ring stretch vibrations, and the other one at $1043-1030 \mathrm{~cm}^{-1}$ was assigned to stretch vibrations of the C-O bond [27]. Hydrolysable tannins, gallo- and ellagitannins, present one characteristic strong band at 1731-1704 $\mathrm{cm}^{-1}$ and another at $1325-1317 \mathrm{~cm}^{-1}$. The former was assigned to the stretching vibration of the carbonyl function, and the latter to the symmetric stretching of the $\mathrm{C}-\mathrm{O}$ bond of the ester function [28-29]. Ellagitannins showed the mentioned characteristic bands of vegetable tannins (1615-1606, 1518-1507, 1452-1446, 1211-1196, and 1043-1030 $\mathrm{cm}^{-1}$ ) and characteristic bands of the hydrolysable chemical class (1731-1704 $\mathrm{cm}^{-1}$ and 1325-1317 $\mathrm{cm}^{-1}$ ) [27]. From the above results, it was determined that the form of tannin obtained in jasmine tea and jasmine petal tea was a kind of hydrolyzable tannin.

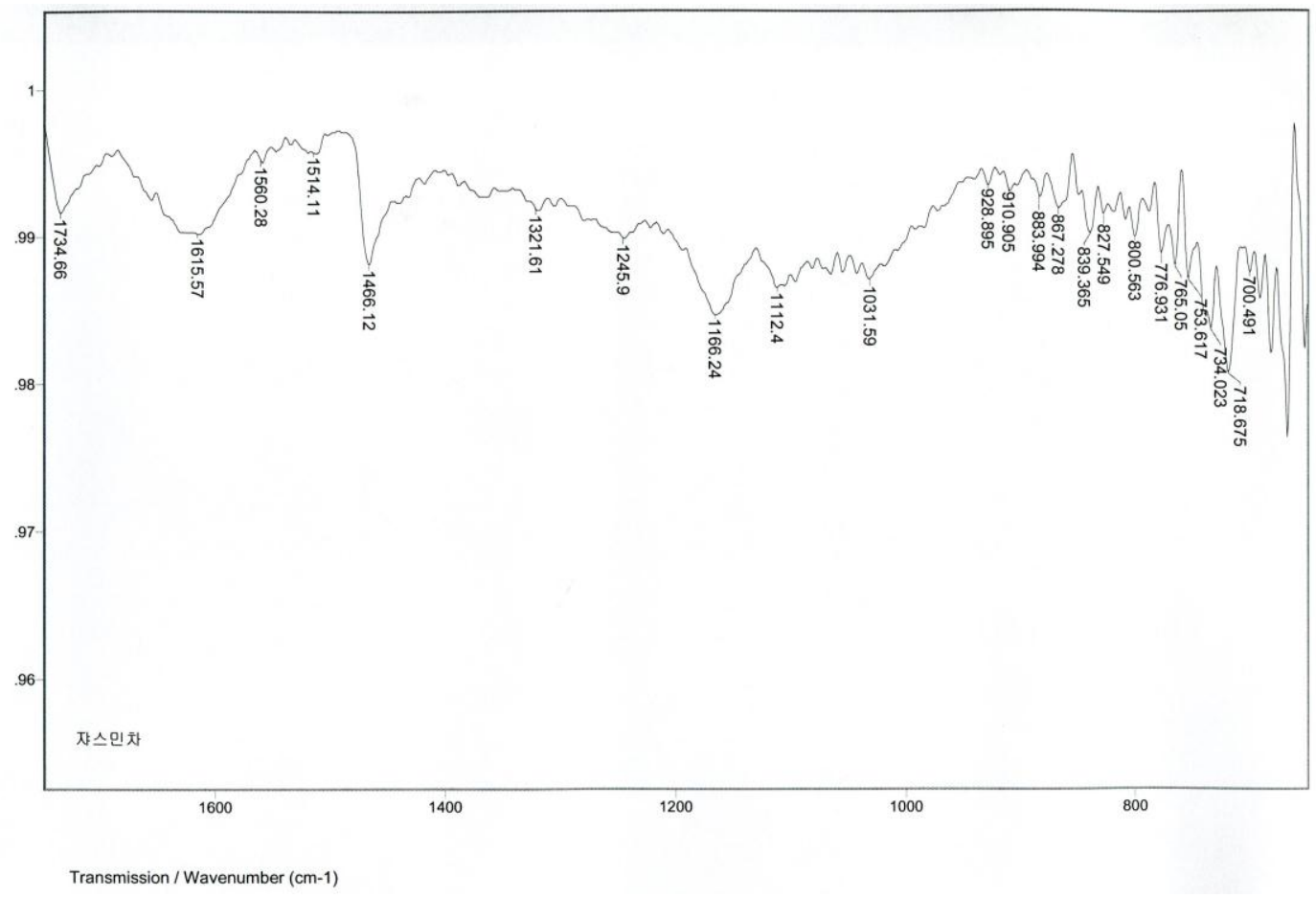

(A) 


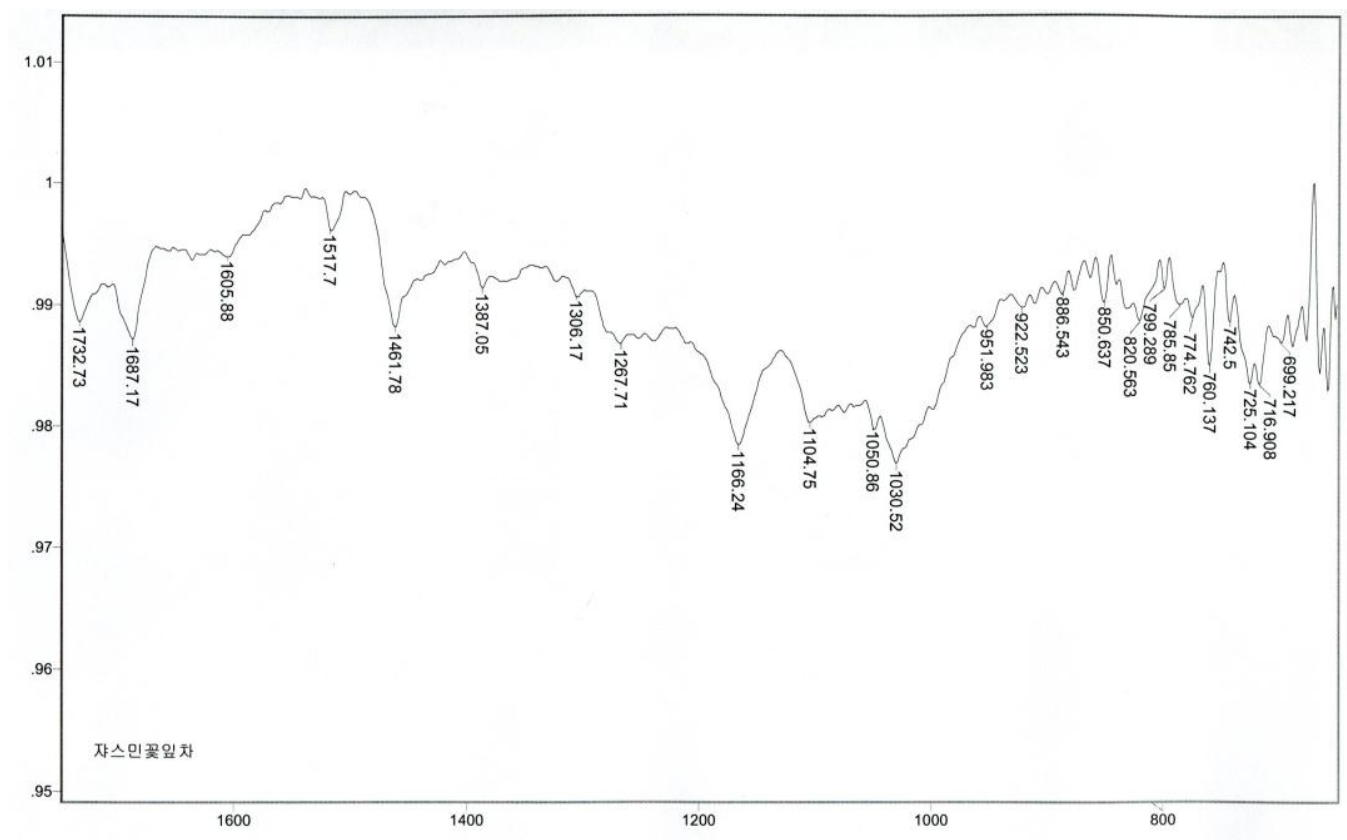

Transmission / Wavenumber (cm-1)

(B)

Figure 2. FT-IR of Jasmine Tea (A) and Jasmine Petal Tea (B)

Table 3. FT-IR Characteristic Bands of Jasmine Tea and Jasmine Petal Tea

\begin{tabular}{c|c|c}
\hline \multicolumn{3}{c}{ Main Vibrational Bands of Jasmine Tea and Jasmine Petal Tea } \\
(Wave Numbers, $\mathrm{cm}^{-1}$, Intensity) \\
\hline Jasmine Tea & Jasmine Petal Tea & $\begin{array}{c}\text { Marker Bands } \\
\text { Spectral Intervals }\end{array}$ \\
\hline & & Tannin Compounds $^{\mathrm{a}}$ \\
\hline 1615.57 & 1605.99 & $1615-1606$ \\
\hline 1511.87 & 1516.94 & $1518-1507$ \\
\hline 1032.76 & & $1452-1446$ \\
\hline & 1030.52 & $1211-1196$ \\
\hline 1734.66 & 1732.73 & Hydrolysable Tannins \\
\hline 1318.97 & & 1731.1704 \\
\hline
\end{tabular}

${ }^{\mathrm{a}}$ : Collected data 


\subsection{Antimicrobial Activity of Dyed Fabrics}

The antimicrobial activity on $S$. aureus of silk fabrics dyed with the jasmine tea extracts and jasmine petal tea extracts are given in Table 4. As shown in this table, silk fabrics dyed with jasmine tea extracts appeared to have significantly high antimicrobial activity of $99.9 \%$, regardless of dyeing concentration. In contrast, no antimicrobial activity was observed for all silk fabrics dyed with jasmine petal tea extracts. The antimicrobial activity on K. pneumoniae of silk fabrics dyed with the jasmine tea extracts and jasmine petal tea extracts are provided in Table 5. As shown in this table, silk fabrics dyed with stock solution and one-half-fold diluted solution had a significantly high antimicrobial activity of $99.9 \%$. In contrast, no antimicrobial activity was observed from all silk fabrics dyed with jasmine petal tea extracts, similar to the findings for $S$. aureus.

Table 4. Bacteriostatic Reduction (\%) against S. aureus of Silk Fabrics Dyed with Jasmine Tea Extracts and Jasmine Petal Tea Extracts

\begin{tabular}{c|c|c|c|c|c|c|c}
\hline & Blank & \multicolumn{3}{|c|}{ Jasmine Tea } & \multicolumn{3}{c}{ Jasmine Petal Tea } \\
\cline { 3 - 8 } & & 1 & $1 / 2$ & $1 / 4$ & 1 & $1 / 2$ & $1 / 4$ \\
\cline { 3 - 8 } & & 99.9 & 99.9 & 99.9 & 0 & 0 & 0 \\
\hline Bacteriostatic Reduction (\%) & - & 9 & & & & & \\
\hline
\end{tabular}

Table 5. Bacteriostatic Reduction (\%) against $K$. pneumoniae of Silk Fabrics Dyed with Jasmine Tea Extracts and Jasmine Petal Tea Extracts

\begin{tabular}{c|c|c|c|c|c|c|c}
\hline & Blank & \multicolumn{3}{|c|}{ Jasmine Tea } & \multicolumn{3}{c}{ Jasmine Petal Tea } \\
\cline { 3 - 8 } & & 1 & $1 / 2$ & $1 / 4$ & 1 & $1 / 2$ & $1 / 4$ \\
\cline { 3 - 8 } & & 99.9 & 99.9 & 0 & 0 & 0 & 0 \\
\hline Bacteriostatic Reduction (\%) & - & & & & & \\
\hline
\end{tabular}

\subsection{Antimicrobial Activity of Dyeing Fabrics after Irradiation}

Antimicrobial activity on $S$. aureus of silk fabrics dyed with the jasmine tea extracts and jasmine petal tea extracts after irradiation for 20 hours are given in Table 6. As shown in this table, the bacteriostatic reduction of silk fabrics dyed with stock solution, one-half-fold diluted solution, and quarter-fold diluted solution after 20 hours of irradiation was $99.9 \%$, 98.\% and 93.3\%, respectively. From these results, it is shown that silk fabrics dyed with jasmine tea extracts present high antimicrobial activity over $93.3 \%$. Antimicrobial activity on $K$. pneumoniae of silk fabrics dyed with the jasmine tea extracts after 20 hours of irradiation is presented in Table 7. As shown in this table, the bacteriostatic reduction of silk fabric dyed with stock solution after 20 hours of irradiation was 40.6\%, whereas no antimicrobial activity was observed from silk fabrics dyed with one-half-fold diluted solution and quarter-fold diluted solution after 20 hours of irradiation.

Table 6. Bacteriostatic Reduction (\%) against S. aureus of Silk Fabrics Dyed with Jasmine Tea Extracts and Jasmine Petal Tea Extracts after Irradiation

\begin{tabular}{l|l|l}
\hline & Blank & Jasmine Tea \\
\hline
\end{tabular}




\begin{tabular}{c|c|c|c|c}
\hline & & 1 & $1 / 2$ & $1 / 4$ \\
\hline \hline Bacteriostatic Reduction (\%) & - & 99.9 & 98.3 & 93.3 \\
\hline
\end{tabular}

Table 7. Bacteriostatic Reduction (\%) against $K$. pneumoniae of Silk Fabrics Dyed with Jasmine Tea Extracts and Jasmine Flower Tea Extracts after Irradiation

\begin{tabular}{c|c|c|c|c}
\hline & Blank & \multicolumn{3}{|c}{ Jasmine Tea } \\
\cline { 3 - 5 } & & 1 & $1 / 2$ & $1 / 4$ \\
\hline \hline Bacteriostatic Reduction (\%) & - & 40.6 & 0 & 0 \\
\hline
\end{tabular}

\subsection{Antimicrobial Activity of Dyeing Fabrics after Dry Cleaning}

The antimicrobial activity on $S$. aureus of silk fabrics dyed with the jasmine tea extracts after dry cleaning are provided in Table 8 . As shown in this table, the bacteriostatic reduction of silk fabrics dyed with jasmine tea extract was significantly high $(99.9 \%)$ as before dry cleaning, regardless of the dyeing concentration. Antimicrobial activity on K. pneumoniae of silk fabrics dyed with the jasmine tea extracts after dry cleaning are given in Table 9. As shown in this table, bacteriostatic reduction of silk fabrics dyed with stock solution and one-half-fold diluted solution after dry cleaning had a significant antimicrobial activity of $99.9 \%$, similar to before dry cleaning.

Table 8. Bacteriostatic Reduction (\%) against S. aureus of Silk Fabrics Dyed with Jasmine Tea Extracts and Jasmine Petal Tea Extracts after Dry Cleaning

\begin{tabular}{c|c|c|c|c}
\hline \multirow{2}{*}{} & \multirow{2}{*}{ Blank } & \multicolumn{3}{|c}{ Jasmine Tea } \\
\cline { 3 - 5 } & & 1 & $1 / 2$ & $1 / 4$ \\
\hline \hline Bacteriostatic Reduction (\%) & - & 99.9 & 99.9 & 99.9 \\
\hline
\end{tabular}

Table 9. Bacteriostatic Reduction (\%) against $K$. pneumoniae of Silk Fabrics Dyed with Jasmine Tea Extracts and Jasmine Petal Tea Extracts after Dry Cleaning

\begin{tabular}{c|c|c|c|c}
\hline \multirow{2}{*}{} & \multirow{2}{*}{ Blank } & \multicolumn{3}{|c}{ Jasmine Tea } \\
\cline { 3 - 5 } & & 1 & $1 / 2$ & $1 / 4$ \\
\hline \hline Bacteriostatic Reduction (\%) & - & 99.9 & 99.9 & 0 \\
\hline
\end{tabular}

\section{Conclusion}

The purpose of this study was to develop a natural functional material with insecticidal effects and antimicrobial activity for use in emotional robots. Part of the study involved determining the antimicrobial activity of silk fabric dyed with jasmine tea and jasmine petal 
tea. The total phenol content was measured to determine the concentrations of phenol components, known to be antimicrobial compounds, contained in jasmine tea and jasmine petal tea. UV-Vis analysis and FT-IR analysis were also used. The results of these experiments are as follows:

1. The total phenol content was higher in jasmine tea than in jasmine petal tea.

2. The ethanol extraction solution of jasmine tea and jasmine petal tea appeared at absorption maxima, $\lambda$ max, at $272 \mathrm{~nm}$ and $277 \mathrm{~nm}$ ( $\lambda$ max of tannin is 272-285), respectively, by UV-Vis analysis.

3. Jasmine tea and jasmine petal tea showed three main common absorption bands in the $1750-700 \mathrm{~cm}^{-1}$ range: $1615-1606,1518-1507$, and $1043-1030 \mathrm{~cm}^{-1}$ by FT-IR analysis. From these results, it was determined that the form of tannin obtained in jasmine tea and jasmine petal tea was a kind of hydrolysable tannin.

4. Silk fabrics dyed with jasmine tea extracts appeared to have quite high antimicrobial activity of $99.9 \%$ with respect to $S$. aureus, regardless of dyeing concentration. Silk fabrics dyed with stock solution and one-half-fold diluted solution of jasmine tea extracts presented a significantly high antimicrobial activity of $99.9 \%$ for $K$. pneumoniae. In contrast, no antimicrobial activity was observed from all silk fabrics dyed with jasmine petal tea extracts, regardless of the form of bacteria tested.

5. Silk fabrics dyed with jasmine tea extracts demonstrated high antimicrobial activity of $93.3 \%$ on S. aureus after 20 hours, regardless of dyeing concentration. Only silk fabric dyed with the stock solution of jasmine tea extracts appeared to have any antimicrobial activity, of $40.6 \%$, on $K$. pneumoniae, after 20 hours of irradiation.

6. Silk fabrics dyed with jasmine tea extract had significant antimicrobial activity of $99.9 \%$ on $S$. aureus, similar to its properties before dry cleaning, regardless of the dyeing concentration. Silk fabrics dyed with stock solution and one-half-fold diluted solution of jasmine tea extracts after dry cleaning had significant antimicrobial activity of $99.9 \%$ on $K$. pneumoniae, similar to their properties before dry cleaning.

\section{Acknowledgements}

This paper is the product of research that was conducted with the support of the Korea Research Foundation with the resources of the Government (Ministry of Education, Science, and Technology) in 2013 (NRF-2013R1A2A2A04014808).

\section{References}

[1] T. Tanaka, "Tanaka $\square$ s Cyclopedia of Edible Plants of the Word”, Keigaku Pub. Co., Tokyo (1976).

[2] S. Shobana, and K. A. Naidu, "Prostaglandins, Leukotrienes and Essential Fatty Acids", vol. 62, (2000), pp. 107-110.

[3] Y. S. Velioglu, G. Mazza, L. Gao, and B. D. Oomah, "Journal of Agricultural and Food Chemistry", vol. 46, (1998), pp. 4113-4117.

[4] W. Zheng, and S. Y. Wang, "Journal of Agricultural and Food Chemistry", vol. 49, (2001), pp. 5165-5170.

[5] S. Inouye, T. Takizawa, and H. Yamaguchi, "Journal of Antimicrobial Chemotherapy", vol.47, (2001), 565573.

[6] D. J. Charles, “Antioxidant properties of spices, herbs and other sources”, Springer, New York (2013).

[7] W. J. Craig, “Am. J. Clin. Nutr. “, vol. 70, (1999), pp. 491-499.

[8] A. Starway, "Attenuative Medicine: A Guide to Natural Therapies", Penguin Book Ltd., Hamondworth, New York, (1982), pp. 18-32.

[9] V. E. Tyler, "The Honest Herbal”, 3rd ed. Haworth Press Inc, Binghamton, New York, (1993), pp. 57-73.

[10] K. Ramakrishnan, S. R. Selve and R. Shubha, "Indian Chemical Engineering", vol. 48, (2006), pp. 88-93.

[11] T. Okuda and H. Ito, "H. Molecules", vol. 16, (2011), pp. 2191-2217. 
[12] H. Akiyama, K. Fujii, O. Yamasaki, T. Oono and K. Iwatsuki, “J. Antimicrob. Chemother.”, vol. 48, (2001), pp. $487-491$.

[13] A. Banso and S. O. Adeyemo, “Afr. J. Biotechnol.”, vol. 15, (2007), pp. 1785-1787.

[14] R. Amarowicz, M. Naczk and F. Shahini, "J. Am. Oil Chem. Soc.”, vol. 77, (2000), pp. 957-961.

[15] G. V. Smirnova, G. I. Vysochina, N. G. Muzyka, Z. Samoilova, T. A. Kukushkina and O. N. Oktabr'skii, Appl. Biochem. Microbiol., vol. 45, (2009), pp. 638-641.

[16] H. Hoste, F. Jackson, S. Athanasiadou, S. M. Thamsborg and S. O. Hoskin, "Trends Parasitol", vol. 22, (2006), pp. 253-261.

[17] M. A. Alonso-Diaz, J. F. J. Torres-Acosta, C. A. Sandoval-Castro, H. Hoste, A. J. Aguilar-Caballero and C. M. Capetillo-Leal, Anim. Feed Sci. Technol., vol. 141, (2008), pp. 36-38.

[18] M. P. Ayres, T. P. Clausen, S. F. MacLean, A. M. Redman and P. B. Reichardt, "Ecology", vol. 78, (1997), pp. 1696-1712.

[19] R. V. Barbehenn, A. Jaros, G. Lee, C. Mozola, Q. Weir and J. P. Salminen, "Vet. Parasitol.”, vol. 55, (2009), pp. 297-304.

[20] P. Buzzini, P. Arapitsas, M. Goretti, E. Branda, B. Turchetti, P. Pinelli, F. Ieri and A. Romani, "Mini-Rev. Med. Chem.", vol. 8, (2008), pp. 1179-1187.

[21] T. Okuda and H. Ito, "H. Molecules", vol. 16, (2011), pp. 2191-2217.

[22] P. Arapitsas, "Food Chem.", vol. 135, (2012), pp. 1708-1717.

[23] A. Zang, Q. Y. Zhu, Y. S. Luk, K. Y. Ho, K. P. Fung and Z. Y. Chen, "Life Science”, vol. 61, (1997), pp. 383-394.

[24] W. Si, J. Gong, R. Tsao, M. Kalab, R. Yang, and Y. Yin, "Journal of Chromatography A", vol. 1125, (2006), pp. 204-210.

[25] S. R. Uhl, "Handbook of spices, seasoning, and flavoring", Lancaster, Pa: Technomic Publishing (2000).

[26] J. H. Sul, "A Study on the Tannin Treatment of Silk Fabrics", Doctoral Thesis, Pusan National University (1994).

[27] L. Falcao and M. E. M. Araujo, "Journal of Cultural Heritage”, vol. 14, (2013), pp. 499-508.

[28] A. Edelmann and B. Lendl, “J. Am. Chem. Soc.”, vol. 124, (2002), pp. 14741-14747.

[29] K. Fernandez and E. Agosin, “J. Agric, Food Chem.”, vol. 55, (2007), pp. 7294-7300.

\section{Authors}

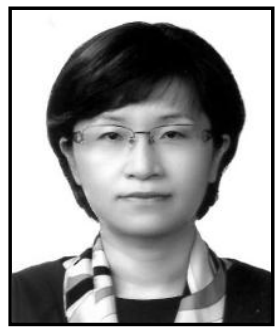

Jin-Soun Jung, Professor, Dept. of Fashion Design, Semyung University, Jecheon, Korea; Bachelor of Home Economics from Pusan National University, Pusan, Korea; Master of Home Economics from Pusan National University, Pusan, Korea; PhD in Science from Pusan National University, Graduate School

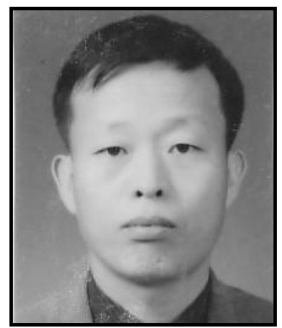

Bo-Hee Lee, Professor, Dept. of Electrical Engineering, Semyung University, Jecheon, Korea; Bachelor of Electronic Engineering from Inha University, Incheon, Korea; Master of Automation Engineering from Inha University, Incheon, Korea; $\mathrm{PhD}$ in Automation Engineering from Inha University, Graduate School 


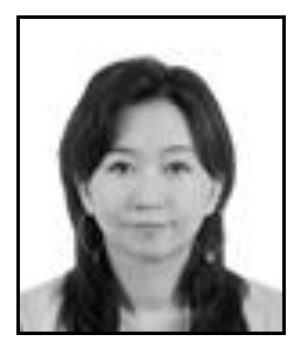

Keum Hi Mun, Professor, Dept. of Industrial Design, Semyung University, Jecheon, Korea; Bachelor of Industrial Design in Seoul National University, Seoul, Korea; Master of Industrial Design in Hongik University, Seoul, Korea

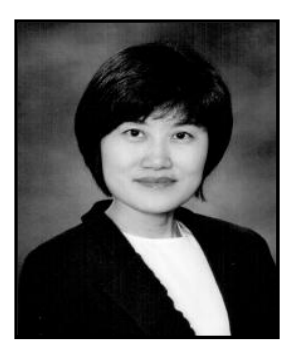

Ja-Young Kwon, Professor, Dept. of Social Welfare, Semyung University, Jecheon, Korea; Bachelor of Social Work from Ewha Womans University, Seoul, Korea; Master of Social Work from Ewha Womans University, Seoul, Korea; Ph D in Social Welfare from Ewha Womans University, Graduate School 